\title{
From positive to colossal negative thermal expansion in a novel family of bimetallic imidazolates
}

\author{
S. Burazer ${ }^{1}$, L. Horák ${ }^{1}$, Y. Filinchuk ${ }^{2}$, M. Dopita1, R. Černý ${ }^{3}$, J. Popović ${ }^{4}$ \\ ${ }^{1}$ MFF, Charles University, Ke Karlovu 5, Prague, CZ, \\ ${ }^{2} I C M N$, Université catholique de Louvain, Place L. Pasteur 1, Louvain-la-Neuve, BE, \\ ${ }^{3} D Q M P$, University of Geneva, Quai Ernest-Ansermet 24, Geneva, CH, \\ ${ }^{4}$ Ruđer Bošković Institute, Bijenička 54, Zagreb, HR
}

\section{Sanja.Burazer@irb.hr}

\begin{abstract}
Materials with negative thermal expansivity (NTE) attracts great attention of scientists because they can be combined with numerous materials with positive thermal expansion (PTE) in order to prepare a composite material with a tailored coefficient of thermal expansion, namely, zero expansion. This allows decreasing a performance deterioration caused by large difference in expansion coefficients.[1] Among numerous metal organic frameworks (MOFs), zeolitic imidazolate frameworks (ZIFs) are highly popular because of a possibility to combine electronic properties of the transition metal ions with structural features of zeolites. They possess large porosity, therefore alkali metals that form dense and hypercoordinated structures stayed out of the focus for its synthesis. On the other hand, magnesium imidazolate have porous structure, as well as its borohydride(s). Although the preparation of manganese imidazolates is challenging, probably due to the difficulties of formation of non-distorted tetrahedral $\mathrm{Mn}^{2+}-4 \mathrm{~N}$ geometry preferable in ZIFs, similarity of magnesium and manganese borohydrides was reason to try synthesis with both metals and compare the results.
\end{abstract}

Mechanochemical reactions of alkali metal imidazolates and magnesium or manganese borohydride gave novel bimetallic imidazolates $\mathrm{AMIm}_{3}(\mathrm{~A}=\mathrm{Na}, \mathrm{K} ; \mathrm{M}=\mathrm{Mg}, \mathrm{Mn})$ whose crystal structures have been solved from synchrotron radiation X-ray powder diffraction (PXRD) data using global optimization in program FOX [2]. Pores of 30-35 $\AA^{3}$ (5-6 \% of the unit cell volume) are incorporated in all structures. Detailed study of temperature-aided structural and microstructural changes, obtained from the synchrotron in situ HT-PXRD data, gave deeper understanding of crystallization processes in borohydride-imidazolate system and have elucidated mechanisms of the reactions which occurs during mechanochemical synthesis and thermal treatment of these systems.

Extensive study of thermal expansion properties of a series of isostructural compounds $\mathrm{AMIm}_{3}(\mathrm{~A}=\mathrm{Na}, \mathrm{K} ; \mathrm{M}=\mathrm{Mg}, \mathrm{Mn})$ revealed a common behaviour characteristic for a structural type. However, very interesting drastic changes of thermal expansion were noticed when alkali metal imidazolate $(\mathrm{NaIm})$ coexist with compound-of-interest $\left(\mathrm{NaMgIm}_{3}\right)$; volumetric thermal expansion coefficient changes from positive $\alpha_{\mathrm{V}}=35 \times 10^{-6} \mathrm{~K}^{-1}$ to colossal negative values $\alpha_{\mathrm{V}}=-460 \times 10^{-6} \mathrm{~K}^{-1}$ and linear thermal expansion changes from $\alpha=34 \times 10^{-6} \mathrm{~K}^{-1}$ to $\alpha=-210 \times 10^{-6} \mathrm{~K}^{-1}$ (Figure 1). This is caused by coherent intergrowth, lattice mismatch, a tensile strain and microstructural properties [3] of mentioned phases and leaves a possibility of design of the material with zero thermal expansivity.

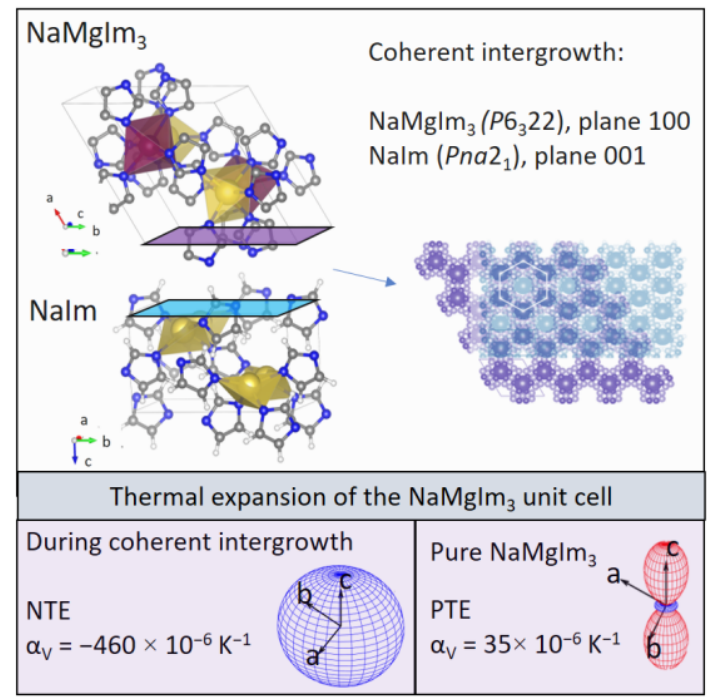

Figure 1. Change of coefficient of thermal expansion of $\mathrm{NaMgIm}_{3}$ from colossal negative during coherent intergrowth between planes $100\left(\mathrm{NaMgIm}_{3}\right)$ and $001(\mathrm{NaIm})$ to positive for pure $\mathrm{NaMgIm}_{3}$ 
[1] Ren, Z.; Zhao, R.; Chen, X.; Li, M.; Li, X.; Tian, H.; Zhang, Z.; Han, G. (2018) Nat. Commun. 123, 1638.

[2] Favre-Nicolin, V.; Černý, R. (2002) J. Appl. Crystallogr. 35, 734-743.

[3] Matěj, Z.; Kužel, R.; Nichtová, L. (2010) Powder Diffr. 25, 125-131.

Keywords: structure determination from X-ray powder diffraction; synchrotron radiation; negative thermal expansion; coherent intergrowth; microstructure

The research was supported by OP RDE project No. CZ.02.2.69/0.0/0.0/18_053/0016976 International mobility of research, technical and administrative staff at the Charles University.

The financial support of the SNSF project (SCOPES) "Metal-Hydride Organic Frameworks (HOF) - new solids for gas adsorption and separation" is acknowledged. 